\title{
Fourier transform plasmon resonance spectrometer using nanoslit-nanowire pair
}

\author{
Cite as: Appl. Phys. Lett. 114, 251101 (2019); doi: 10.1063/1.5092517 \\ Submitted: 11 February 2019 . Accepted: 27 May 2019 . \\ Published Online: 24 June 2019
}

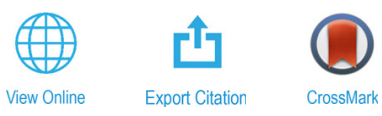

Doolos Aibek Uulu, 'Timur Ashirov, (D) Nahit Polat, ${ }^{2}$ (D) Ozan Yakar, ${ }^{2}$ Sinan Balci, ${ }^{2, a)}$ (D) and Coskun Kocabas ${ }^{3, a)}$ (D)

AFFILIATIONS

${ }^{7}$ Institute of Materials Science and Nanotechnology, Bilkent University, Ankara 06800, Turkey

${ }^{2}$ Department of Photonics, Izmir Institute of Technology, Izmir 35430, Turkey

${ }^{3}$ School of Materials, University of Manchester, Oxford Rd, Manchester M13 9PL, United Kingdom

a) Authors to whom correspondence should be addressed: sinanbalci@iyte.edu.tr and coskun.kocabas@manchester.ac.uk

\begin{abstract}
In this paper, we present a nanoscale Fourier transform spectrometer using a plasmonic interferometer consisting of a tilt subwavelength slit-nanowire pair on a metallic surface fabricated by the focused ion beam microfabrication technique. The incident broadband light strongly couples with the surface plasmons on the gold surface, and thus, surface plasmon polaritons (SPPs) are generated. The launched SPPs interfere with the incident light and generate high contrast interference fringes in the nanoslit. The transmitted SPPs through the metal nanoslit can decouple into free space and are collected by an objective in the far field. The spectroscopic information of the incidence light is obtained by fast Fourier transform of the fringe pattern of the SPPs. In our design, there is no need for a bulky dispersive spectrometer or dispersive optical elements. The dimension of the spectrometer is around $200 \mu \mathrm{m}$ length. Our design is based on inherent coherence of the SPP waves propagating through the subwavelength metal nanoslit structures etched into an opaque gold film.
\end{abstract}

Published under license by AIP Publishing. https://doi.org/10.1063/1.5092517

Recent advances in nanoscale fabrication techniques, chemical synthesis, and patterning of nanomaterials have provided us with new techniques and tools to measure optical properties of light at the nanoscale. ${ }^{1}$ Light can be tightly confined to nanoscale volumes by using surface plasmon polaritons (SPPs), ${ }^{2}$ which were first predicted by Ritchie in 1957. ${ }^{3}$ Ritchie demonstrated that the energy losses of fast electrons $(\sim 40 \mathrm{keV})$ when passing through a thin metal foil are due to the surface and volume plasmons. ${ }^{3}$ SPPs are transverse surface electromagnetic waves propagating along a metal-dielectric interface. The intensity of SPPs is very high at the metal surface and decreases exponentially with the distance from the metal surface. The discovery of surface plasmon assisted optical transmission through subwavelength holes drilled in opaque metal films has garnered interest in SPPs. ${ }^{1}$ The SPP enabled concentration of light into nanoscale volumes has greatly enhanced the interaction of light with matter and boosted the performance of nanophotonic devices owing to the enhancement of the field intensity compared to the incident light. ${ }^{4}$ For example, by using gold nanoparticle core and dye doped silica shell nanoparticles, surface plasmon assisted lasing ( $50 \mathrm{~nm}$ in size coherent light source) has been demonstrated. ${ }^{5}$ In other studies, owing to the interference of plasmon waves on the photosensitive polymer surface, researchers have proposed and experimentally demonstrated large area SPP enabled subwavelength lithography. ${ }^{6,7}$ In another study, an on-chip optical plasmonic spectrometer based on holographic metallic gratings for coupling and decoupling free-space radiation has been demonstrated. ${ }^{8}$

Interference of SPPs and free space light in the nanoslit-groove pairs is very attractive to measure the coherence and phase of plasmon waves. $^{9-14}$ However, there has not been any report on the application of the nanoslit-nanowire pair as a plasmon spectrometer. In the old spectrometers, a prism was used to disperse light, which was first discovered by Newton. ${ }^{15}$ However, most of the modern spectrometers use diffraction gratings to disperse light into its constituent colors. Recently, colloidal quantum dot based spectrometers have been shown where a two-dimensional absorptive filter array consisting of colloidal quantum dots has been used to measure the light spectrum based on the wavelength multiplexing principle. ${ }^{16}$ Here in this study, we show a Fourier transform plasmon resonance (FTPR) nanospectrometer based on interference of the plasmon wave in the subwavelength metal nanoslit, see Fig. 1. To couple incident light to surface plasmons, a metal nanowire or ridge is deposited next to the nanoslit by using focused ion beam (FIB) deposition. The launched SPPs between the metal and dielectric interface interfere with the incident light and thus generate high contrast interference fringes of SPP waves which travel through the nanoslit. The spectroscopic information of the incidence 

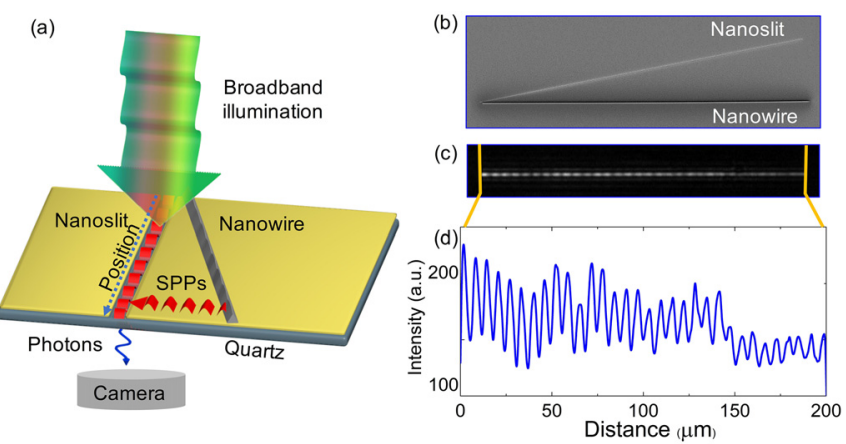

FIG. 1. (a) Schematic drawing of the Fourier transform plasmon resonance (FTPR) nanospectrometer consisting of subwavelength slit-nanowire plasmonic interferometer fabricated by focused ion beam (FIB) etching and metal nanowire (or ridge) deposition. Broadband incident visible light strongly couples with the surface plasmons on the metal nanowire surface and generates surface plasmon polaritons, i.e., photon to polariton conversion. The SPP waves interfere with the incident light and yield the interference pattern in the nanoslit. Depending on the optical path difference between the nanowire and the nanoslit, destructive or constructive interference is observed. The nanoslit void is empty. The transmitted SPPs decouple to free space light, i.e., polariton to photon conversion. The plasmon interference pattern in the spatial domain, which is called an interferogram, is generated in the nanoslit. The fast Fourier transform (FFT) of the interfering plasmon waves gives the wavelength of the plasmon wave. (b) Scanning electron microscopy image of a typical subwavelength slit-nanowire pair forming a plasmonic interferometer on a gold surface. The length of the slit is typically several micrometers long $(\sim 200 \mu \mathrm{m})$ and $\sim 100 \mathrm{~nm}$ wide. (c) The interference pattern of the waves captured by a charged coupled device (CCD) camera. (d) Different optical path lengths introduce a phase shift between the incoming light and SPP wave in the nanoslit. Hence, the line scan across the interference pattern reveals the dark and bright regions, indicating destructive and constructive interference of waves, respectively.

light is obtained by fast Fourier transform (FFT) of the fringe pattern of the SPPs. Owing to the coherence of SPP waves on the metal surface, the interference pattern can be generated in the nanoslit. ${ }^{14}$ The most important advantage of our spectrometer is that the dimension of the spectrometer is very small, around $200 \mu \mathrm{m}$, and hence, light matter interaction in small regions can be extensively studied. For example, the dynamics of the plasmon-exciton coupling in small regions can be studied $^{17}$ and dark excitons can be probed via near field coupling to surface plasmon polaritons in the nanoslit-groove pair as well.

Opaque gold films (around $200 \mathrm{~nm}$ thick) were deposited on a quartz substrate by thermal evaporation. The nanoslits and Pt nanowires on the gold film were fabricated by using Focused Ion Beam (FIB), which is a versatile technique for fabricating very small nanostructures with a sub10 $\mathrm{nm}$ resolution by using ion beam etching and metal deposition. ${ }^{19}$ The FIB is very similar to a scanning electron microscope (SEM), but the FIB system uses $\mathrm{Ga}^{+}$ions rather than electrons in the SEM system. Alternatively, electron beam lithography can be used instead of FIB to eliminate metal ion contamination in sample fabrication. ${ }^{20}$ The voltage and current of the FIB during nanoslit fabrication were $30 \mathrm{kV}$ and $28 \mathrm{pA}$, respectively. The nanoslit void and metal surface are left empty, and thus, SPPs propagate at an air-metal interface. However, during electron beam assisted metal nanowire fabrication, the voltage and current were $5 \mathrm{kV}$ and $1.6 \mathrm{nA}$, respectively. It should be noted here that ridge geometry offers better excitation efficiency than groove geometry, and thus, metal ridges or nanowires are used in this study to couple incident light to surface plasmons. ${ }^{21}$ The lengths of the nanoslit and metal nanowire were around $150 \mu \mathrm{m}$. The optimum height of the nanowire is around $80 \mathrm{~nm}$ as shown in Fig. 2. Indeed, the Michelson contrast has been calculated for a variety of nanowire heights from the interference fringes, and a height of $80 \mathrm{~nm}$ has been shown to have the maximum Michelson contrast. The ridge is tilted by $5^{\circ}$ with respect to the main axis of the nanoslit. Therefore, the distance (optical path) between the nanoslit and the ridge is linearly controlled. A $635 \mathrm{~nm}$ diode laser with an output power of $4.5 \mathrm{~mW}$ was used. The SPPs were excited by the laser on the metal nanowire. The incoming light polarization is p-polarized. The electric field vector is perpendicular to the nanowire axis. The momentum mismatch between the incoming light and SPPs was compensated by the nanowire. The coupled SPPs propagating on the gold surface interfere with incoming light in the nanoslit, and hence, the fringe pattern is formed in the nanoslit. In fact, the SPPs go through the nanoslit and decouple from the gold surface and are reconverted back into the photons. A $20 \times$ objective efficiently collects the scattered photons and sends them to a CCD camera. The CCD camera was cooled to $-76^{\circ} \mathrm{C}$ during imaging to reduce dark currents. The interference fringes were imaged using an inverted microscope (Nikon) equipped with the above optical components. More details about the imaging setup are provided in SFig. 1. Three dimensional finite difference time domain simulations were performed using a commercial package (Lumerical FDTD).

Figure 1 shows the schematic representation of the FTPR nanospectrometer proposed in this study. Incident light cannot excite the surface plasmons on the flat metal surfaces owing to the momentum mismatch between the surface plasmons and incident light. ${ }^{22}$ The momentum mismatch can be compensated by using a prism or a grating. In the case of grating, the wave vector of the SPP can be calculated as

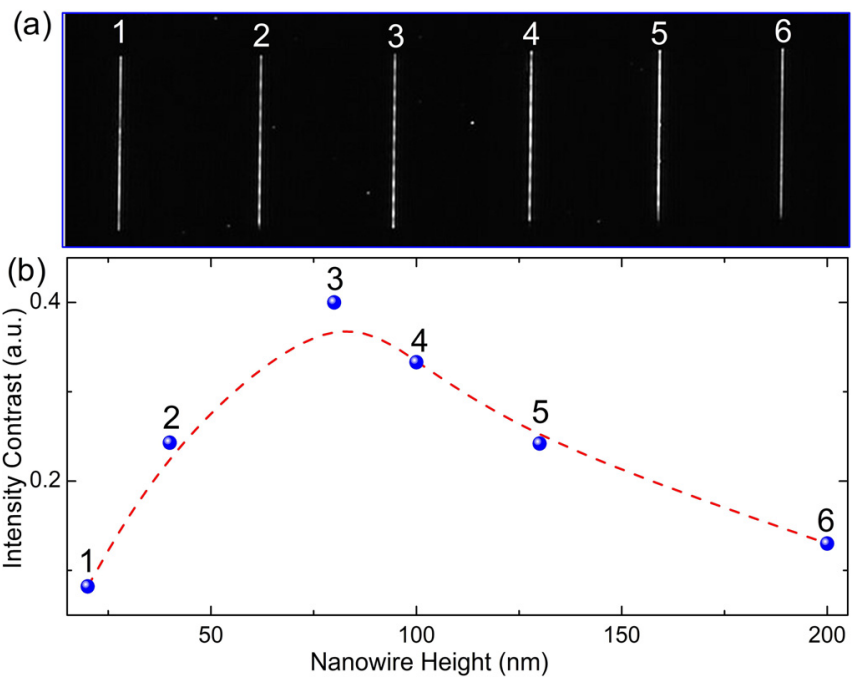

FIG. 2. Optimization of the metal nanowire height. (a) A charged coupled device (CCD) image of the interference patterns with varying metal nanowire heights. The height of the nanowire increases from the first interference pattern (1) to the last interference pattern (6). (b) The Michelson contrast [Michelson contrast $=\left(I_{\max }\right.$ $\left.\left.-I_{\min }\right) /\left(I_{\max }+I_{\min }\right)\right]$ of the interference pattern as a function of the nanowire height. The maximum Michelson contrast has been observed in the third interference pattern, and hence, in most of the samples studied, the height of the metal nanowires has been chosen to be around $80 \mathrm{~nm}$. 


$$
k_{s p p}=k_{0} \sqrt{\frac{\epsilon_{m} \epsilon_{d}}{\epsilon_{m}+\epsilon_{d}}}=k_{0} \sqrt{\epsilon_{d}} \sin \theta \pm m \frac{2 \pi}{\Lambda} \quad(m= \pm 1,2 \ldots),
$$

where $k_{0}=2 \pi / \lambda_{0}$ is the wave vector of the incident light, $\lambda_{0}$ is the wavelength of the incident light, $\epsilon_{m}$ is the dielectric constant for the metal, $\epsilon_{d}$ is the dielectric constant for the dielectric medium, $\Lambda$ is the grating period, $\theta$ is the angle of the incident light, $k_{s p p}$ is the wave vector of the SPPs, and $m$ is the diffraction order of the grating. Like a prism, a grating can excite the surface plasmons as well. Therefore, the metal ridge couples the incident light (photon) to surface plasmons (plasmon), and SPPs (polariton) are generated on the gold surface and they propagate at the interface between the air and gold film, Fig. 1. The propagating SPPs interact with the incoming light in the nanoslit, and interference fringes are formed in the nanoslit. The interferogram in the spatial domain is converted to the frequency domain, which gives information about the frequency of the incoming light, Fig. 1. The intensity of the interferogram decays exponentially with the optical path difference since the decay length of SPP, $L_{D}$, is proportional to $1 / \operatorname{Im}\left(\mathrm{k}_{\mathrm{spp}}\right) .{ }^{14} \mathrm{In}$ fact, the plasmon wave vector has real and imaginary parts: $k_{s p p}=\operatorname{Re}\left(k_{s p p}\right)+\operatorname{Im}\left(k_{s p p}\right)=\operatorname{Re}\left(k_{s p p}\right)+i /\left(2 L_{D}\right){ }^{23}$

The interference pattern in the nanoslit shows the dark and bright regions representing destructive and constructive interference of waves, respectively, which yields the wave vector of SPPs. Figure 1(b) represents the SEM image of the nanoslit-ridge pair fabricated on the gold surface using FIB milling and deposition techniques. The length of the structure is around $200 \mu \mathrm{m}$. The height of the Pt ridge is around $80 \mathrm{~nm}$. In this study, we have used a ridge instead of a groove in the gold surface since it has been found that the excitation efficiency of a single ridge is higher than that of a single groove. ${ }^{21}$ The metal nanowire is slightly tilted with respect to the main axis of the nanoslit. It should be noted that in classical Fourier transform spectroscopy, the intensity variation as a function of optical path difference $(\delta)$ is measured by using a Michelson interferometer. ${ }^{24}$ In fact, the moving mirror in the Michelson interferometer gives the optical path difference. Here, in this study, the tilted nanowire gives the optical path
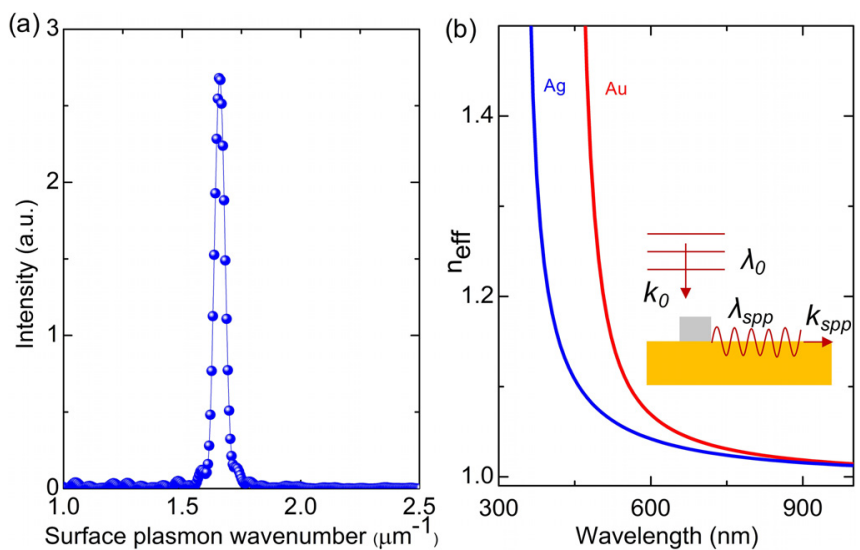

FIG. 3. (a) Fourier transform of the interference fringes gives the wave vector of SPPs. (b) Effective indexes ( $\left.n_{\text {eff }}\right)$ of gold and silver as a function of the incident wave wavelength. With the help of the metal nanowire on the gold surface, surface plasmon polaritons are excited. The excited transverse plasmon polaritons propagate on the gold surface. The momentum mismatch between the free space light (photons) and SPPs on the gold surface is compensated by the metal ridge. difference. The interference pattern in the nanoslit was imaged in the far field by a CCD camera and is reported in Fig. 1(c). The intensity line profile across the nanoslit main axis indicates that the intensity of the wave decreases, Fig. 1(d). This is due to the fact that the nanowirenanoslit distance increases from left to right in the image and SPP intensity decreases away from the nanowire. Also note that the SPP propagation length, proportional to $1 / \operatorname{Im}\left(\mathrm{k}_{\mathrm{spp}}\right)$, increases with the large real (negative) and small imaginary parts of the relative permittivity of the metal.

(a)

(b)

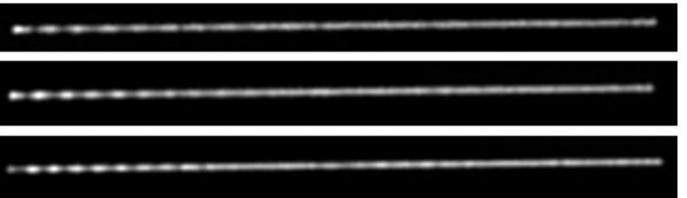

(c)
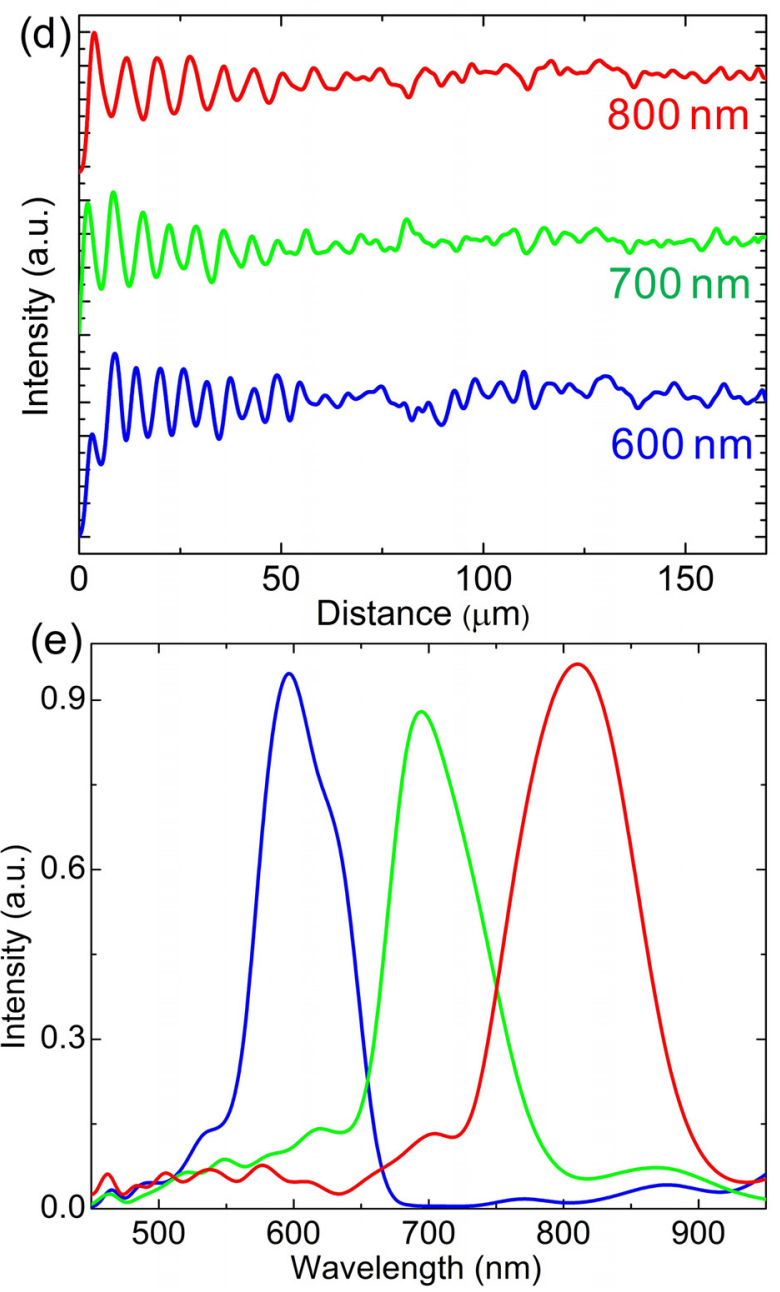

FIG. 4. FTPR nanospectrometer. [(a)-(c)] Interference fringes of SPPs in the nanoslit with varying wavelengths of the incident light. (d) Line scans across the interference pattern, i.e., interference pattern in the spatial domain. (e) Wavelength of the incident light can be obtained from the Fourier transform of the interference fringes in the spatial domain. 

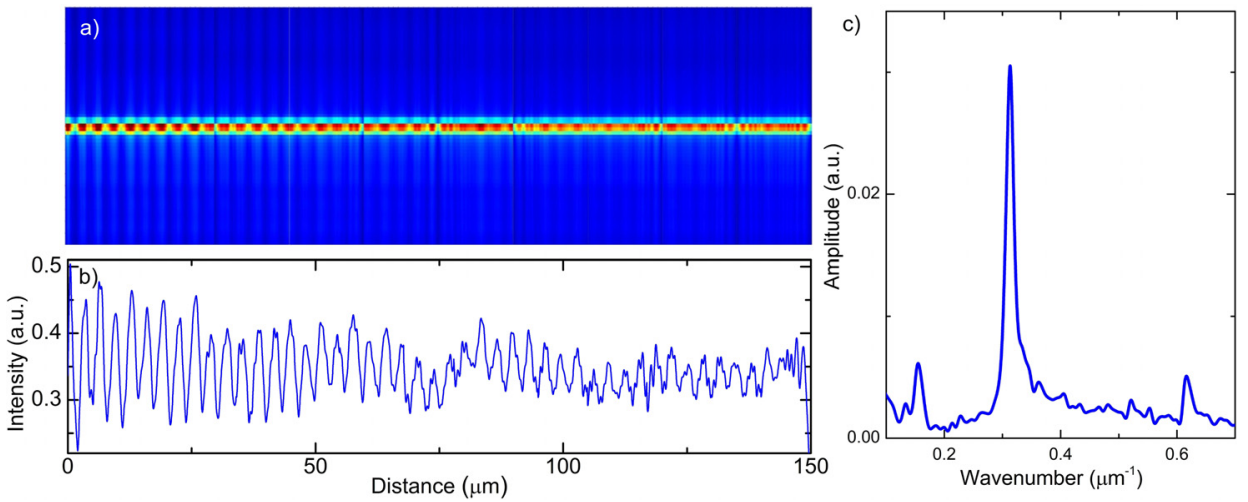

FIG. 5. Three dimensional finite difference time domain simulations of the nanowire-nanoslit pair. (a) Interference pattern in the nanoslit. Simulation window consists of the subwavelength slit-nanowire plasmonic interferometer. The length of the nanoslit in the simulation window is $150 \mu \mathrm{m}$. Incident visible light strongly couples with the surface plasmons on the nanowire and generates surface plasmon polaritons. The SPP waves interfere with the incident light and yield the interference pattern in the nanoslit. The transmitted SPPs decouple to free space light, i.e., polariton to photon conversion. (b) Electric field intensity emerging from the nanoslit. Owing to the decay of the surface plasmon polaritons, the Michelson contrast of the interference pattern decreases toward the end of the nanoslit. (c) Fourier transform of the interference fringes in (b) yields the plasmon wave vector.

In order to understand the fringe pattern observed in Fig. 1(b), we can safely assume two plane waves of the same frequency $E_{r}$ and $E_{s}$ impinging on the ridge and slit, $E_{r}=E_{01} \cos \left(k x_{r}-\omega t+\varphi_{1}\right)$ and $E_{s}$ $=E_{01} \cos \left(k x_{s}-\omega t+\varphi_{1}\right)$, where $k=2 \pi / \lambda, \varphi$ is the phase of the wave at the source when $t=0$, and $x$ is the optical path difference. ${ }^{26}$ At the nanowire, some portion of the $E_{r}$ will be coupled to surface plasmons. At the slit, the beams will interfere and total irradiance $I=\epsilon_{0} c\langle E . E\rangle$, where total electric field $E=E_{r}+E_{s}, c$ is the speed of light in vacuum, and $\epsilon_{0}$ is the permittivity of free space. The resulting irradiance at the slit $I=\epsilon_{o} c\left\langle E_{r} \cdot E_{r}+E_{s} \cdot E_{s}+2 E_{s} \cdot E_{r}\right\rangle=I_{r}+I_{s}+2\left(I_{r} I_{s}\right)^{1 / 2} \cos (\delta)$, where $\delta$ is the phase difference between $E_{r}$ and $E_{s}$. When $\cos (\delta)$ is +1 and -1 , constructive and destructive interference generates the maximum irradiance, $I_{\max }=I_{r}+I_{s}+2\left(I_{r} I_{s}\right)^{1 / 2}$, and minimum irradiance, $I_{\min }=I_{r}$ $+I_{s}-2\left(I_{r} I_{s}\right)^{1 / 2}$, respectively. ${ }^{26}$ Notify that $I_{\min }$ can only be zero when $I_{r}=I_{s}$. This is not possible in our case since only some portion of the incoming beam at the nanowire will couple to surface plasmons. ${ }^{12}$ Therefore, in Fig. 1(b) and most of the fringe patterns observed in this study, $I_{\min }$ is not zero. Then, the visibility of the fringe pattern sometimes also called the Michelson fringe visibility can be expressed as $I_{\text {max }}-I_{\text {min }} / I_{\text {max }}+I_{\text {min. }}{ }^{26}$

Fast Fourier transform of the interference pattern in the spatial domain given in Fig. 1(c) generates the intensity profile in the wave vector domain in Fig. 3(a). The SPP wave vector, $k_{s p p}$, can be deduced from this graph. Since $k_{s p p}=n_{e f f} k_{0}=n_{e f f} 2 \pi / \lambda_{0}\left(n_{e f f}\right.$ is the effective index), the wavelength or the frequency of the incident light can be calculated by knowing $k_{s p p}$, see Fig. 3(a) for the effective indexes of gold and silver. In a similar way, the plasmon wavelength can be expressed as $\lambda_{s p p}=2 \pi / \operatorname{Re}\left(k_{s p p}\right)=\lambda_{0} / n_{\text {eff. }}{ }^{14}$ Also note that the SPP propagation length, proportional to $1 / \mathrm{Im}\left(\mathrm{k}_{\mathrm{spp}}\right)$, increases with the large real (negative) and small imaginary parts of the relative permittivity of the metal, ${ }^{25}$ see Fig. 3(b). To show the wavelength selectivity of the FTPR nanospectrometer, by using the same nanoslit-nanowire pair, the wavelength of the incident light is varied. In Fig. 4, the incident wavelengths are 600,700 , and $800 \mathrm{~nm}$. The fringe pattern in the nanoslit and their intensity profile for different wavelengths are shown, Fig. 4. The Fourier transform of the interference fringes in the spatial domain generates the intensity of the incident light in the frequency or wavelength domain, Fig. 4(e). Furthermore, the validity of the experimental results was confirmed by performing three dimensional finite difference time domain simulations using a commercially available package (Lumerical FDTD), Fig. 5 and SFig. 6. In Fig. 5, the simulation window contains the subwavelength slit-nanowire plasmonic interferometer. The length of the nanoslit in the simulation window is $150 \mu \mathrm{m}$. Figure 5(b) demonstrates the interference pattern in the nanoslit. Due to the decay of the SPPs, the Michelson contrast in the interference pattern decreases toward the end of the nanoslit. The Fourier transform of the interference fringes produces the plasmon wave vector, see Fig. 5(c).

In conclusion, we present a fast Fourier transform based nanospectrometer called a Fourier transform plasmon resonance spectrometer, which is based on the interference of surface plasmon polariton waves and free space light in the subwavelength metal nanoslit. The SPPs between the metal and dielectric interface are launched by the Pt nanowire. The optical path between the Pt ridge and the nanoslit was controlled by tilting the Pt nanowire by $5^{\circ}$ with respect to the nanoslit main axis. Owing to the optical path difference between the incoming light and SPPs, constructive and destructive interference patterns in the nanoslit have been observed. The transmitted SPPs decouple from the metal surface and are collected by an objective in the far field and eventually detected by a standard CCD sensor. The spectroscopic information of the incidence light is obtained by Fourier transform of the fringe pattern of the SPPs. Our design is based on inherent coherence of the SPP waves propagating through the subwavelength metal slits perforated on a gold surface. The dimension of the spectrometer is around $200 \mu \mathrm{m}$ long. The proposed technique can be used for measuring optical properties of light at the nanoscale dimension ${ }^{27}$ and for sensing the refractive variation at the nanoscale as a nanospectrometer. ${ }^{28}$ In addition, the nanospectrometer can be used to measure plasmon-exciton hybridization in a small region. ${ }^{17}$

See the supplementary material for more detailed descriptions regarding the (i) experimental setup for measuring the wavelength of 
the incident light using the FTPR spectrometer, (ii) surface plasmon polariton decay length with the ridge-slit distance, (iii) atomic force microscopy image of the Pt nanowire, (iv) comparison of Fourier transform plasmon resonance and Fourier transform infrared spectrometers, and (v) three dimensional finite difference time domain simulations of the nanowire-nanoslit pair.

This research was partially supported by the European Research Council (ERC) Consolidator (Grant No. ERC-682723 SmartGraphene) and also partially supported by the Scientific and Technological Research Council of Turkey (TUBITAK) (Grant No. 114F052).

\section{REFERENCES}

${ }^{1}$ T. W. Ebbesen, H. J. Lezec, H. F. Ghaemi, T. Thio, and P. A. Wolff, Nature 391(6668), 667-669 (1998).

${ }^{2}$ W. L. Barnes, A. Dereux, and T. W. Ebbesen, Nature 424(6950), 824-830 (2003).

${ }^{3}$ R. H. Ritchie, Phys. Rev. 106, 874 (1957).

${ }^{4}$ R. A. Pala, J. White, E. Barnard, J. Liu, and M. L. Brongersma, Adv. Mater. 21(34), 3504 (2009).

${ }^{5}$ M. A. Noginov, G. Zhu, A. M. Belgrave, R. Bakker, V. M. Shalaev, E. E. Narimanov, S. Stout, E. Herz, T. Suteewong, and U. Wiesner, Nature 460(7259), 1110-U1168 (2009).

${ }^{6}$ Z. W. Liu, Q. H. Wei, and X. Zhang, Nano Lett. 5(5), 957-961 (2005).

${ }^{7}$ X. W. Guo, J. L. Du, Y. K. Guo, and J. Yao, Opt. Lett. 31(17), 2613-2615 (2006).

${ }^{8}$ Y. Tsur and A. Arie, Opt. Lett. 41(15), 3523-3526 (2016).
${ }^{9}$ V. V. Temnov, K. Nelson, G. Armelles, A. Cebollada, T. Thomay, A. Leitenstorfer, and R. Bratschitsch, Opt. Express 17(10), 8423-8432 (2009).

${ }^{10}$ B. Ung and Y. L. Sheng, Opt. Express 15(3), 1182-1190 (2007).

${ }^{11}$ X. Zeng, H. F. Hu, Y. K. Gao, D. X. Ji, N. Zhang, H. M. Song, K. Liu, S. H. Jiang, and Q. Q. Gan, Sci. Rep. 5, 12665 (2015).

${ }^{12}$ D. Morrill, D. F. Li, and D. Pacifici, Nat. Photonics 10(10), 681-687 (2016).

${ }^{13}$ C. H. Gan, G. Gbur, and T. D. Visser, Phys. Rev. Lett. 98(4), 043908 (2007).

${ }^{14}$ H. F. Schouten, N. Kuzmin, G. Dubois, T. D. Visser, G. Gbur, P. F. A. Alkemade, H. Blok, G. W. Hooft, D. Lenstra, and E. R. Eliel, Phys. Rev. Lett. 94(5), 053901 (2005).

${ }^{15}$ I. Newton, Am. J. Phys. 61(2), 108-112 (1993).

${ }^{16}$ J. Bao and M. G. Bawendi, Nature 523(7558), 67 (2015).

${ }^{17}$ S. Balci and C. Kocabas, Opt. Lett. 40(14), 3424-3427 (2015).

${ }^{18}$ Y. Zhou, G. Scuri, D. S. Wild, A. A. High, A. Dibos, L. A. Jauregui, C. Shu, K. De Greve, K. Pistunova, A. Y. Joe, T. Taniguchi, K. Watanabe, P. Kim, M. D. Lukin, and H. Park, Nat. Nanotechnol. 12(9), 856 (2017).

${ }^{19}$ K. Celebi, J. Buchheim, R. M. Wyss, A. Droudian, P. Gasser, I. Shorubalko, J. I. Kye, C. Lee, and H. G. Park, Science 344(6181), 289-292 (2014).

${ }^{20}$ M. Horak, K. Bukvisova, V. Svarc, J. Jaskowiec, V. Krapek, and T. Sikola, Sci. Rep. 8, 9640 (2018).

${ }^{21}$ H. T. Liu, P. Lalanne, X. Y. Yang, and J. P. Hugonin, IEEE J. Sel. Top. Quantum Electron. 14(6), 1522-1529 (2008).

${ }^{22}$ S. Balci, C. Kocabas, S. Ates, E. Karademir, O. Salihoglu, and A. Aydinli, Phys. Rev. B 86(23), 235402 (2012).

${ }^{23} \mathrm{H}$. Raether, Springer Tracts in Modern Physics (Springer, 1988), Vol. 111, pp. 1-133.

${ }^{24} \mathrm{P}$. R. Griffiths, Science 222(4621), 297-302 (1983).

${ }^{25}$ W. L. Barnes, J. Opt. A 8(4), S87-S93 (2006).

${ }^{26}$ E. Hecht, Optics (Pearson, Boston, 2017).

${ }^{27}$ T. Bauer, S. Orlov, U. Peschel, P. Banzer, and G. Leuchs, Nat. Photonics 8(1), 23-27 (2014).

${ }^{28}$ O. Yavas and C. Kocabas, Opt. Lett. 37(16), 3396-3398 (2012). 